\title{
DOLOR FÍSICO, ATENCIÓN Y ESQUEMA CORPORAL: CONSIDERACIONES FENOMENOLÓGICAS SOBRE LA NATURALEZA DEL DOLOR
}

\section{PHYSICAL PAIN, ATTENTION AND BODY SCHEMA: SOME PHENOMENOLOGICAL CONSIDERATIONS ABOUT THE NATURE OF PAIN}

\author{
Paula Diaz Romero ${ }^{1}$ \\ Instituto de Humanidades-CONICET (Argentina)
}

Recibido: 16-9-2015

Aceptado: 29-3-2016

\begin{abstract}
Resumen: Con el objetivo de esclarecer algunos de los aspectos más significativos de los análisis fenomenológicos del dolor físico, en este trabajo pretendo mostrar que la fenomenología del dolor es un caso particular de análisis dentro del marco general de la fenomenología de la corporeidad. Para ello consideraré, por un lado, la relación entre las modalidades atencionales y la aparición del dolor y, por otro lado, la relación entre la aparición del dolor y la perturbación del esquema corporal. Por último, a fin de justificar mi tesis, trataré la relación entre atención y esquema corporal.
\end{abstract}

Palabras Claves: dolor; atención; esquema corporal; cuerpo; fenomenología.

\begin{abstract}
With the aim of elucidating some important aspects of the phenomenological analysis of physical pain, I will show in this paper that Phenomenology of Pain constitutes a singular case within the general context of the Phenomenology of Body. In order to show that thesis, I will consider, first, the relationship between attentional modes and the birth of pain and, second, the relationship between birth of pain and body schema disturbance. Finally, with the objective of justifying mi own thesis, I will consider the relationship between attention and body schema.
\end{abstract}

Key-Words: pain; attention; body schema; body; phenomenology.

1. (pauladiazromero@gmail.com) Licenciada en Filosofía por la Universidad Nacional de Córdoba. Actualmente doctoranda en Filosofía en la Universidad Nacional de Córdoba con beca del Consejo Nacional de Investigaciones Científicas y Técnicas (CONICET)- Argentina. Miembro de los grupos de investigación Cuerpo, sujeto y afectividad: interlocutores, antecedentes y contexto de diálogo (SECYT-UNC) y Proyecto de Reactualización contemporánea de la Fenomenología: Fenomenología de la Corporeidad (FONCYT-Argentina), ambos bajo la dirección de Ariela Battán Horenstein. 


\section{Introducción}

A mediados del siglo XX, la fenomenología francesa da un giro de lo trascendental a lo corporal. Este giro, disimulado por el ambiente cartesiano que insiste en atraer a muchos fenomenólogos como J.P. Sartre y M. Henry, se materializa en la obra de M. Merleau-Ponty bajo el concepto conciencia encarnada.

El concepto de conciencia encarnada reconoce el carácter fundamental del cuerpo y la situación y establece el ambiente apropiado para el desarrollo de aquellas investigaciones que pretenden deshacerse de los vestigios del dualismo mente-cuerpo. Incluso en la actualidad, esta impronta de volver hacia el cuerpo para dar cuenta del conocimiento del mundo ha trascendido los límites de las investigaciones puramente fenomenológicas, posibilitando diálogos interdisciplinarios.

En este contexto, temas como la percepción, la motricidad, la emoción, el dolor, etc., pueden ser objetos de un análisis minucioso de su estructura fenoménica y eidética, y develar los vínculos intencionales que se establecen en la relación de esta corporeidad y el mundo. Un caso que pone en evidencia la existencia encarnada es la experiencia del dolor físico. El dolor se convierte en un caso paradigmático, pues, se experiencia como un fenómeno lindante entre lo físico y lo psíquico, lo objetivo y lo subjetivo. ${ }^{2}$

2. Esta complejidad es la que le otorga al fenómeno su carácter enigmático y atractivo. El dolor físico, agudo y crónico, se presenta como un problema para la fisiología y para la psicología. Tal como sostiene J. Choza: "Si se tiene en cuenta que el dolor es, ante todo, un hecho en el acontecer vital de una intimidad, en cuanto intimidad consciente y corpórea, y que la intimidad consciente es inaccesible a la fisiología, se echa de ver inmediatamente que el análisis experimental fisiológico accede a la dimensión más extrínseca del dolor" (Choza J., "Dimensiones antropológicas del dolor", Anuario Filosófico, vol. 10, n² 2, 1997, 40). En el mismo sentido puede decirse que "(d)esde el análisis fenomenológico exclusivamente se puede establecer una clasificación del dolor según su intensidad y según la tonalidad afectiva que lleva consigo, pero desde esta posición metodológica resulta difícil establecer la naturaleza, el origen y el sentido del dolor en la economía de la vida humana. Por ejemplo, se hace difícil determinar si el dolor es una sensación o un sentimiento, y se hace muy difícil establecer con exactitud la estructura de la subjetividad humana y la incidencia del dolor en sus diversos elementos estructurales" (Ibídem, 41).

Teniendo en cuenta la insuficiencia explicativa de estos dos "polos" de análisis, es importante aclarar que el presente estudio fenomenológico reconoce la limitación de la perspectiva de la primera persona, sobre la cual trata la fenomenología tal como la presenta Choza. Sin embargo, aquí se toma partido por una fenomenología de la existencia (siguiendo a Merleau-Ponty) que constituye un punto medio y superador de las dimensiones objetivas y subjetivas, tanto para el análisis de la experiencia del cuerpo, como la del dolor. De hecho, en Fenomenología de la Percepción, Merleau-Ponty sostiene que "(l)o que permite ligar uno con otro, lo "fisiológico" con lo "psíquico", es justamente que, reintegrados a la existencia, ya no se distinguen como el orden del en sí y el orden del para sí y que los dos están orientados hacia un polo intencional o hacían un mundo" (Merleau-Ponty, M. Fenomenología de la percepción; Ed. FCE; México; 1957, p. 95). Incluso, "el hombre, concretamente tomado, no es un psiquismo unido

Thémata. Revista de Filosofía No54 (2016) pp.: 175-190. 
Esta característica fundamental del fenómeno permite tratarlo a partir de un marco general denominado Fenomenología de la corporeidad. Esta perspectiva reconoce -y esta es su tesis central- que el sujeto de adscripción del dolor es, en todos los casos, el cuerpo: el cuerpo vivido a partir del cual es posible toda experiencia del mundo. Sin dudas, en este contexto general, el caso particular de la investigación fenomenológica del dolor ha comenzado a cruzar la barrera del mero "prolegómeno» hacia un análisis maduro de la naturaleza del fenómeno doloroso. ${ }^{3}$ Claros ejemplos de esto son los estudios más recientes de Agustín Serrano de Haro, Saulius Geniusas, Carmen López Sáenz, Drew Leder. ${ }^{4}$ La intención de muchas de estas investigaciones es lograr una cierta independencia de los marcos más generales de análisis, constituyendo un ámbito de investigación autónomo, con la intención de que sea posible hablar de una Fenomenología del dolor.

Con el objetivo de esclarecer algunos de los aspectos más significativos de los análisis fenomenológicos del dolor físico, en este trabajo pretendo mostrar que la fenomenología del dolor es un caso particular de análisis dentro del marco general de la fenomenología de la corporeidad. Para ello consideraré, por un lado, la relación entre las modalidades atencionales y la aparición del dolor y, por otro lado, la relación entre la aparición del dolor y la perturbación del esquema corporal. Luego, destacaré la existencia de una relación entre la perturbación de la atención y la perturbación del esquema corporal. Por último, voy a presentar las razones por las cuales la

a un organismo, sino este vaivén de la existencia que en un momento se deja corporizar y en otro va hacia actos personales" (Ídem).

3. Geniusas, S. "On naturalism in pain research: a phenomenological critique", Metodo. International Studies in Phenomenology and Philosophy, 2013, Vol. vol. 1, n. 1.

4. Serrano de Haro, A.: "Defensa de la perspectiva fenomenológica en el análisis del dolor" en Pensar la compasión, Ed. Universidad Pontifica Comillas, Reflexiones Comillas, Filosofía, 1, Año 59, N. 959, 2009. P 161-172. A propósito de la fenomenología del dolor, Crítica, Año 62, №. 981, 2012. Y "Atención y dolor. Análisis Fenomenológico", José Ortega y Gasset/José Gaos/Joaquín Xirau/ Leopoldo-Eulogio Palacios/Agustín Serrano de Haro, Cuerpo vivido. Madrid, Encuentro, 2010. Geniusas, S.: "On naturalism in pain research: a phenomenological critique", Metodo. International Studies in Phenomenology and Philosophy, 2013, Vol. vol. 1, n. 1. Y "The origins of the phenomenology of pain: Brentano, Stumpf and Husserl", Continental Philosophy Review, Vol. 47, Issue 1, pp. 1-17, 2014. Leder, D. The absent body, The Univessity of Chicago Press, Chicago, United States of America, 1990. López Sáenz, C. "Hermenéutica del cuerpo doliente-dolido desde la fenomenología del sentir" en Investigaciones Fenomenológicas, Vol. monográfico 2: "Cuerpo y alteridad”, 2010. 
fenomenología del dolor físico es un caso paradigmático dentro de lo que se conoce como fenomenología de la corporeidad.

\section{Atención}

Uno de los aspectos más notables que involucra la vivencia del dolor es, sin dudas, la inminente atención que recae sobre él. Sea de forma parcial o completa, siempre que siento un daño físico mi atención se ve perturbada. Por esta razón considero fundamental iniciar estas reflexiones a partir de la recuperación del análisis que Agustín Serrano de Haro lleva a cabo en Atención y Dolor. Análisis fenomenológico, en donde se establecen las relaciones esenciales entre dolor-conciencia-atención-cuerpo. En dicha obra, Serrano de Haro resalta el valor significativo del caso del dolor físico para los estudios fenomenológicos por ser una experiencia global que involucra el interés de la conciencia y devela su corporeidad. Se destaca en dicho trabajo que «el dolor en el cuerpo se dibuja (...) como un acontecimiento especialmente revelador de estructuras fenomenológicas». ${ }^{5}$ Esto es posible ya que «[e]l dolor del cuerpo guarda, pues, con la atención de la conciencia vínculos estrechos e inmediatos, pero a la vez flexibles y reveladores, que sin duda merecen el rigor del análisis y el beneficio de una cierta ordenación». ${ }^{6}$

Una de las primeras cuestiones que señala el autor es una correspondencia entre las modificaciones atencionales que sufre la conciencia ante la inminente aparición del dolor y una primera clasificación del dolor que se establece por la intensidad del dolor sufrido y la atención que reclama tal dolor. El interés está puesto en describir cómo se modifica el foco de atención de la conciencia sobre el mundo, tanto cuando el dolor se presenta espontánea y drásticamente así como cuando el dolor es constante, crónico y se funde con el horizonte de sentido de mi vida.

El primer rasgo relevante y esencial del dolor físico es su «demostración de influjo y poderío a la hora de dominar la vida de la atención». ${ }^{7}$ Sin embargo, existen grados más altos y bajos de dolor que corresponden a la comprensión cuatripartita de las modalidades atencionales propuestas por E. Husserl. A partir de esta idea se establece una primera ordenación de las dolencias que se corresponden con estas modalidades:

5. Serrano de Haro, A. "Atención y dolor. Análisis Fenomenológico" en José Ortega y Gasset/ José Gaos/Joaquín Xirau/ Leopoldo-Eulogio Palacios/Agustín Serrano de Haro, Cuerpo vivido. Madrid, Encuentro, 2010, p. 130/131.

6. Op. Cit., p 124.

7. Ídem.

Thémata. Revista de Filosofía N54 (2016) pp.: 175-190. 


\begin{tabular}{|l|l|}
\hline \multicolumn{1}{|c|}{ Dolencias } & Modalidades atencionales \\
\hline $\begin{array}{l}\text { Dolor que implica la atención } \\
\text { de la conciencia. }\end{array}$ & $\begin{array}{l}\text { Atención como foco de } \\
\text { interés por el cual el dolor } \\
\text { aparece como el presente de } \\
\text { la conciencia. }\end{array}$ \\
\hline $\begin{array}{l}\text { Dolor mantenido en un } \\
\text { segundo plano de interés. }\end{array}$ & $\begin{array}{l}\text { La co-atención, por lo que el } \\
\text { dolor está en el modo de una } \\
\text { co-presencia. }\end{array}$ \\
\hline $\begin{array}{l}\text { Las dolencias latentes } \\
\text { que no interrumpen los } \\
\text { quehaceres de nuestra } \\
\text { atención (molestias } \\
\text { corporales). }\end{array}$ & El fondo u horizonte. \\
\hline $\begin{array}{l}\text { Las dolencias insensibles o } \\
\text { dolencias inconscientes. }\end{array}$ & Margen atencional \\
\hline
\end{tabular}

Los primeros dos niveles de dolor se juegan en el primer plano atencional, mientras que el tercer y cuarto nivel se difuminan en el segundo plano atencional. De todos modos, el poder del dolor excede esta clasificación. Por ejemplo, los dolores fuertes pueden involucrar todas las modalidades atencionales ya que dominan la vida entera de la conciencia copando el foco de la atención, perturbando la co-atención y causando impacto en la desatención. «De modo que aunque el dolor admite distintas valencias atencionales lo peculiar del caso estriba por lo pronto en cómo desde cualquiera de ellas se cierne sobre las otras y concierne a todas aquellas con que convive en la situación global». ${ }^{8}$ La vivencia del dolor tiene la particularidad de destacar la existencia silenciosa del cuerpo, pues, el cuerpo normalmente es un objeto de co-atención, esto es, de una atención paralela a la primaria que recae sobre las cosas o el sector de cosas de mi interés. En un artículo de 1997, Serrano de Haro ya anunciaba este carácter peculiar de la vivencia del dolor: «[1]a tendencia del cuerpo a mantenerse en la esfera de la co-atención, sin caer en completa desatención ni ocupar el centro temático, contrasta con el modo brusco en que él copa la preferencia intencional al suscitarse vivencias de dolor».${ }^{9} \mathrm{Si}$ bien el dolor no puede ser

\section{Ibídem, p.127.}

9. Serrano de Haro, A. "Fundamentación para una fenomenología del cuerpo" en La posibilidad de la fenomenología, Madrid, Ed. Complutense, 1997, p. 189. 
protencionalmente anticipado, existe un "antes inmediato" del aparecer del dolor que el autor describe en términos de conmoción: "se trata de un sobresalto aturdido que interrumpe la continuidad que yo estaba viviendo y que es sentido internamente como una quiebra». ${ }^{10}$ Esta experiencia es lo último que llega a «retenerse y recordarse», por lo cual, en general, no somos conscientes de esta antesala del dolor. Sin embargo, esta "anticipación" no se da en toda experiencia de dolor, por lo cual no la convierte en un momento del dolor sino simplemente de un aparecer eventual anterior a la experiencia dolorosa. Respecto a la temporalidad del dolor, si bien no puedo anticipar protensivamente su aparecer, el dolor siempre dura, es decir, demanda una distensión temporal: un dolor nunca llega e inmediatamente se esfuma.

El dolor producido por una hernia de disco -debido a la compresión de un segmento del nervio ciático o del nervio lumbar- como caso de dolor crónico, puede ser presentado para evidenciar aún más el juego de modalidades atencionales. El dolor de ciático acompaña cada uno de mis movimientos y tareas, sin embargo, ciertas posturas o movimientos bruscos, incluso tensión muscular, pueden lograr que ese dolor que se confunde con mi horizonte de tareas, se patentice repentinamente. A partir del incremento del dolor por un movimiento que acentúa el pinzamiento de la vértebra sobre el nervio, el dolor que acompañaba mi labor silenciosamente puede experienciarse como un dolor repentino y punzante, como un golpe de electricidad que me impide concentrar la atención sobre el trabajo que estoy escribiendo, pues, en el momento en el que estoy logrando concebir una idea, mi existencia corporal opaca mi actividad intelectual, reclamando la atención y el cambio postural de forma urgente. A partir de este ejemplo, considero que se hace claro el carácter fundamental de la vivencia dolorosa que Serrano de Haro pone en los siguientes términos: «el padecimiento doloroso a diferencia de otros estados corporales defectivos cuenta siempre con una focalización que lo ubica en el cuerpo vivido». ${ }^{11}$

Considero importante plantear en este punto una posible distinción de la experiencia del dolor como (i) vivencia del cuerpo y como (ii) percepción del cuerpo. ${ }^{12}$ Con relación a (i) el cuerpo se experiencia como fondo

10. Serrano de Haro, A. A propósito de la fenomenología del dolor, Crítica, Año 62 , №. 981 , 2012, p. 134.

11. Ibidem, p. 142.

12. La distinción trazada no pretende referir a una distinción entre una percepción interna y una percepción externa del mismo. Con el fin de esclarecer esta diferenciación entre dos experiencias del cuerpo, el texto de Jorge Arregui “Cenestesia y cuerpo vivido. ¿Por qué Marcel abandonó sus primeras formulaciones sobre el cuerpo sujeto?”, es de sumo interés. Dice Arregui que "muchas veces se ha planteado la diferencia entre el cuerpo-sujeto y el cuer-

Thémata. Revista de Filosofía No54 (2016) pp.: 175-190. 
que se actualiza ante la irrupción del dolor y que me invita a cambiar la postura irguiéndome "inconscientemente", es decir, el dolor se vive en un plano de conciencia irreflexiva. En cuanto a (ii), el cuerpo puede ser representado como el lugar (topos) desde el cual se irradia el dolor, y en este sentido formar parte de una tematización de la propia corporeidad, de una imagen del cuerpo como cuerpo enfermo, cuerpo herido, etc. Estas son posibilidades de captación de la corporeidad que involucran una dimensión espacial de la experiencia dolorosa. Pues, la experiencia del dolor reclama una cierta organización o re-organización de mi propio cuerpo, esto es, un cambio postural, un abrupto volverme hacia la zona aparentemente afectada, un tocar y presionar la herida en el momento de algidez dolorosa, etc. Sin dudas, en este análisis, el cuerpo tiene la peculiaridad de acompañar a la conciencia como condición cinestésica ${ }^{13}$ de los actos intencionales, pero al no identificarse plenamente con ella, parece quedar supeditado a la misma como un íntimo «objeto» que la acompaña y asiste. En mi opinión, para lograr un análisis de la vivencia del dolor no se debe caer en una captación perceptual del cuerpo (lo cual podría pensarse del análisis de Serrano de Haro). Para ello, el concepto de «esquema corporal» es fundamental, ya que permite comprender mejor la relación entre la atención y los cambios comportamentales ante la aparición del dolor físico.

\section{Esquema corporal}

El caso de la atención sirve de guía para comprender en qué sentido el fenómeno del dolor físico puede ser captado con mayores matices desde una perspectiva encarnada. Como señalé a partir de los ejemplos, el caso del dolor físico despierta un peculiar interés por la capacidad motriz de la corporeidad ya que el dolor no abarca solamente la sensación o percepción interna del daño, ni solamente la captación afectiva del mal. La experien-

po-objeto basándola en la diferencia entre los dos presuntos tipos de percepción que tenemos sobre nuestro cuerpo, oponiendo una percepción interna a una percepción externa del cuerpo propio" (Arregui J. V. "Cenestesia y cuerpo vivido. ¿Por qué Marcel abandonó sus primeras formulaciones sobre el cuerpo sujeto?" Daimon. Revista de Filosofía, n. ${ }^{\circ} 32,2004$, p.148). Arregui señala que el propio G. Marcel cae en esta clasificación en sus primeros escritos. Sin embargo, Marcel avanzaría en su concepción de la corporeidad llegando a la conclusión de que "(j)usto porque es la condición de posibilidad de todo atender y de todo sentir, justo porque goza de una prioridad absoluta, mi cuerpo - el cuerpo propio- no es un objeto, tampoco el de un sentir interno; el cuerpo sujeto no es, pues, de ninguna manera una cosa entre las cosas (Ibidem, 150). Es en el mismo sentido que trazo la distinción entre un cuerpo percibido, objetivado, y un cuerpo vivido.

13. Cfr. Serrano de Haro, A. "Fundamentación para una fenomenología del cuerpo" en La posibilidad de la Fenomenología, Madrid, Ed. Complutense, 1997, p. 194-204.

Thémata. Revista de Filosofía No54 (2016) pp.: 175-190. 
cia del dolor involucra ambas, y supone, además, cambios posturales, cierta gestualidad, etc. Siguiendo el análisis del sentir de L. Landgrebe, se puede afirmar que el sentir dolor implica «un sentirse a sí mismo, porque se trata de una conciencia cinestésica, esto es, una conciencia que implica en sí la conciencia del 'yo me muevo'». ${ }^{14}$

Para dar cuenta de esta característica peculiar del dolor, recuperaré algunos conceptos de Maurice Merleau-Ponty. Si bien el tema del dolor físico no aparece en Fenomenología de la percepción más que mediante referencia a casos puntuales de experiencias de dolor o en relación con experiencias mórbidas, tales referencias resultarán enriquecedoras para las presentes reflexiones. Mediante estas ejemplificaciones de vivencias que no pueden ser concebidas desde un punto de vista reduccionista, como el caso del dolor y la enfermedad, pondré hincapié especialmente en la distinción entre imagen corporal y esquema corporal, con el fin de arrojar luz sobre la dimensión espacial que se presenta en toda vivencia del dolor físico. Según Shaun Gallagher «Merleau-Ponty (1945/1962) utiliza el término esquema corpóreo (traducido como imagen corporal en inglés) para significar un funcionamiento experiencial y dinámico del cuerpo viviente en su entorno. Este esquema incluye una conciencia marginal del cuerpo y opera como un sistema de equivalencias motoras dinámicas que pertenecen al ámbito de los hábitos, antes que al de la elección consciente». ${ }^{15}$ Esta noción de esquema corporal subyace al concepto de conciencia o subjetividad encarnada, pues implica un conjunto de hábitos sedimentados, independientes y previos a toda conciencia reflexiva. ${ }^{16}$ Por el contrario, el concepto de imagen corporal, implica una conciencia reflexiva del cuerpo o, en otros términos, una "representación" del cuerpo. Es en este sentido que podemos afirmar que el cuerpo aparece ante la conciencia como un objeto.

Merleau-Ponty se refiere al caso del dolor a partir de un ejemplo sencillo y puntual. Dice el autor que cuando profiero un juicio del tipo me duele el pie «[1]o que quiero decir es que el dolor señala su lugar, que constituye un 'espacio doloroso'. 'Me duele el pie' no significa 'pienso que mi pie es causa de este mal', sino 'el dolor viene de mi pie', o también 'mi pie padece'». ${ }^{17}$ Para Merleau-Ponty, todo juicio referido a un dolor corresponde a la vivencia de un sufrimiento encarnado. En este sentido, el dolor y la

14. Landgrebe, L. "Principios de la teoría de la sensación", en El camino de la fenomenología, Ed, Sudamericana, Buenos Aries 1963, p. 181.

15. Gallagher, S."Body image and body schema: A conceptual clarification” en Journal of Mind \& Behavior, Autumm,1986, Volume 7, Number 4, p. 542. La traducción es propia.

16. Merleau-Ponty, M.Fenomenología de la percepción, F.C.E., Mexico-Buenos Aires, 1957, Traducción: Emilio Uranga.

17. Op. Cit, p.100.

Thémata. Revista de Filosofía No54 (2016) pp.: 175-190. 
enfermedad involucran experiencias que afectan al hombre completo, es decir, al propio esquema corporal. El golpe del dedo chico del pie tendrá como consecuencia, además de la batería de maldiciones proferidas, una contracción de todas las falanges y una breve renguera (que dependerá de la intensidad del dolor) que requerirá una modificación de mi postura habitual. En este sentido, el esquema corporal refiere a una espacialidad de situación. La situación cambia según aparecen en mi mundo habitual dolencias o enfermedades, ya que estas experiencias implican, en casi todos los casos, que mi campo de significaciones prácticas se vea modificado. Esto sucede, según la opinión de Carmen López Sáenz, porque

(...) el esquema actúa como un arco intencional que permite al cuerpo tener un mundo y, al mismo tiempo, diferenciarse de él gracias a su propia experiencia de la auto-moción corporal. Reparemos en que "auto-movimiento" no es movimiento en un espacio, sino intencionalidad motora, fenomenalización del mundo a través de la espacialización corporal. Es esta intencionalidad la que se pone en juego en la autoconciencia de las afecciones y no la que se distancia de su objeto convirtiéndolo en representación. ${ }^{18}$

Este movimiento permite que el cuerpo propio pueda re-significar su nueva situación, en el caso de la enfermedad, o recomponga la postura, en el caso de la irrupción del dolor. Sin dudas la enfermedad se experimenta como una nueva normatividad para el organismo (un nuevo conjunto de acciones corporales) que se instituye a partir del desajuste entre el cuerpo habitual y cuerpo actual. El cuerpo habitual, entendido como el conjunto de hábitos adquiridos, se disocia del cuerpo actual ante la aparición de ciertas patologías -por ejemplo, el caso del miembro fantasma. En el caso de los dolores crónicos, la irrupción y persistencia del dolor puede llegar a quebrar indefinidamente las dimensiones organizadoras del espacio y el tiempo, dejando aislado a quien lo padece en una vivencia subjetiva intransferible, situación que acompaña cada movimiento del organismo. ${ }^{19} \mathrm{El}$ esquema corporal entra aquí en una crisis de equilibrio, mientras tiende a la reorganización, sin suponer una imagen corporal particular. La imagen

18. López Sáenz, C. "Hermenéutica del cuerpo doliente-dolido desde la fenomenología del sentir" en Investigaciones Fenomenológicas, Vol. monográfico 2: "Cuerpo y alteridad", 2010, p.97.

19. Cf. López Sáenz, C. "Hermenéutica del cuerpo doliente-dolido desde la fenomenología del sentir" en Investigaciones Fenomenológicas, Vol. monográfico 2: "Cuerpo y alteridad”, 2010.

Thémata. Revista de Filosofía No54 (2016) pp.: 175-190. 
del cuerpo solo puede ser considerada, en un segundo momento, como topos de la experiencia.

Un ejemplo muy significativo de disociación entre imagen y esquema corporal es el caso de la algoalucinosis o dolor del miembro fantasma. Se llama miembro fantasma a la sensación de que un miembro amputado continúa unido al cuerpo. Un gran número de casos experimenta esta presencia fantasmagórica como dolorosa. ${ }^{20} \mathrm{El}$ neurólogo V. S. Ramachandran relata en su libro Phantoms in the Brain, distintos casos de experiencias dolorosas del miembro fantasma. Una de las particularidades que destaca es el hecho de que muchos pacientes sientes su miembro paralizado, conservando la misma posición en la que estaba antes de la amputación. Ramachandran ha desarrollado una terapia paliativa para tratar estos casos que resulta casi tan curiosa como el caso del miembro fantasma. La llamada "terapia de la caja espejo» (Mirror Box Therapy) ${ }^{21}$ permite a quienes han sido amputados mitigar el dolor del miembro ausente (aunque la terapia es efectiva solo en el $80 \%$ de los casos). La experiencia con la caja espejo permite al sujeto distender y cambiar la posición del miembro paralizado a partir de la imagen que su miembro sano refleja en el espejo (que esconde, a su vez, al miembro amputado). Este sencillo sistema usa una imagen o una simulación óptica, que logra finalmente una reorganización del esquema corporal. Lo más interesante del caso es el hecho de que el reajuste tiene que ver con la posibilidad de poner en movimiento el miembro fantasma mediante una imagen visual de mi cuerpo que completa la faltante. Quienes logran ver su miembro amputado moviéndose, aunque sean conscientes de que no es más que el reflejo del miembro sano en un espejo, pueden mover el miembro fantasma hasta que el dolor o la incomodidad desaparecen.

Esta terapia ayuda a la reorganización del esquema corporal, y evidencia una relación muy interesante entre la visión, la sensación y el movimiento de la mano. Tanto la experiencia cenestésica como la cinestésica del cuerpo se amalgaman por medio del ver el movimiento sentido de la mano ausente. ${ }^{22} \mathrm{~A}$ su vez, el caso del fantasma señala una ambigüedad:

20. Los síntomas que acompañan al síndrome del miembro fantasma son: dolor, sensación de que el miembro está funcionando normalmente, entumecimiento del miembro amputado, hormigueo, sensación de deformidad, sensación de frio y calor.

21. Ramachandran, V. S. - Blackeslee, S. Phantoms in the Brain, William Morrow and Co., New York, 1998.

22. Los términos de cinestesia (sensación del movimiento del propio cuerpo) y cenestesia (capacidad originaria de sentir el propio cuerpo) son conceptos inseparables. La sensación cenestésica del cuerpo posibilita una experiencia cinestésica particular y, a su vez, las condiciones cinestésicas motivan a una sensación cenestésica diferente. Tanto cinestesias como

Thémata. Revista de Filosofía No54 (2016) pp.: 175-190. 
la ausencia del miembro y la presencia de esta ausencia hace del miembro fantasma una cuasi-presencia. Esta "cuasi-presencia", como la llama Merleau-Ponty, quiebra con las explicaciones de la psicología y la fisiología sobre el caso y habilita un ámbito existencial de análisis que da cuenta de fenómenos de esta naturaleza. A partir de este ejemplo, podemos decir que la experiencia del dolor involucra una conciencia que no puede ser escindida, ni siquiera conceptualmente, de su corporalidad. La noción de conciencia encarnada da cuenta de un ámbito de experiencia que involucra la captación del espacio como campo intencional, como espacio por el cual el cuerpo se mueve y da sentido. En el caso del dolor como vivencia encarnada, altera la situacionalidad global actual. El mundo se ve modificado y no solo perturba la captación atencional, sino que abarca la percepción global del mundo. Ante un dolor dejo de moverme del mismo modo: la postura retraída que adopto ante un dolor de espalda no lo permite, la breve renguera posterior a un golpe en la rodilla me hace perder el equilibrio normal. Desde casos leves -simples molestias corporales- hasta los casos en lo que el dolor paraliza, se patentiza la modificación del esquema corporal y, de este modo, la percepción del espacio y del tiempo.

\section{Atención y esquema}

Un posible enfoque fenomenológico sobre el cuerpo invita a considerarlo como órgano de la percepción. ${ }^{23}$ Según esta interpretación el cuerpo es la «sede de los órganos de los sentidos que, estimulado por factores externos, sirven al conocimiento del medio, y también centro de referencia de las regiones del espacio exterior que se orientan en torno a él (...)». ${ }^{24}$ Según mi parecer, hay en estas palabras una referencia al cuerpo como objeto.

cenestesias son condiciones de posibilidad para toda experiencia. Lo son incluso para la experiencia del propio cuerpo. De hecho, en este plano, el cuerpo no puede ser conocido, pues sería concebido como un objeto más del mundo. Cinestésica y cenestésicamente, el cuerpo es vivido irreflexivamente. Con relación a estos temas puede consultarse la siguiente bibliografía específica: Para el concepto de cinestesia ver Morales Canela, Luis Alberto, "El concepto fenomenológico de cinestesia y la correlación con las secuencias del campo visual: un análisis a las lecciones de Cosa y espacio de 1907”, Eikasia Revista de Filosofía, º 47, enero 2013, Oviedo, pp 749-766. Para el concepto de cenestesia: Arregui J. V. "Cenestesia y cuerpo vivido. ¿Por qué Marcel abandonó sus primeras formulaciones sobre el cuerpo sujeto?” Daimon. Revista de Filosofía, ${ }^{\circ} 32,2004,145-158$.

23. Cfr. Serrano de Haro, A. "Fundamentación para una fenomenología del cuerpo" en La posibilidad de la fenomenología, Madrid, Ed. Complutense, 1997, pp. 185-216. En este escrito Serrano de Haro presenta cuatro direcciones para el estudio fenomenológico del dolor. Para los fines de mi presentación, he decidido destacar solamente dos de ellas.

24. Serrano de Haro, A. "Fundamentación para una fenomenología del cuerpo" en La posibilidad de la fenomenología, Madrid, Ed. Complutense, 1997, p.186.

Thémata. Revista de Filosofía No54 (2016) pp.: 175-190. 
Sin embargo, tal como Serrano de Haro argumenta a lo largo del artículo Fundamentos para una fenomenología del cuerpo, esta concepción puede ser enriquecida si se admite que el cuerpo vivido, como sujeto de experiencia, es parte original de la corriente de vivencia en primera persona y de su dinámica intencional. En este sentido, el cuerpo no es tan solo un objeto referencial en un espacio geométrico, sino que es centro de referencias o punto cero de orientación y anclaje en el mundo. Aquí damos un giro hacia una corporeidad que, a través de su capacidad de moverse en el espacio vivido, abre un mundo de sentido. Como sostiene Merleau-Ponty en muchas ocasiones, el espacio orientado ${ }^{25}$-espacio vivido que no se reduce a sus dimensiones geométricas- y el movimiento son condiciones de posibilidad para la experiencia del mundo. Estos conceptos se encuentran entretejidos en la existencia corporal: es el espacio el que hace posible la acción del sujeto encarnado, pero a su vez, el espacio es orientado solo en la medida en que el sujeto encarnado introduce en él sus coordenadas. El espacio original por el cual el sujeto encarnado se mueve y crea sentido es el esquema corporal que otorga al cuerpo vivido el eje postural y situacional.

Sobre esta misma línea, el cuerpo también puede ser considerado como realidad carencial y necesitada de atención, es decir, como «una amplia gama de sensaciones internas, de afectos y pulsiones sensibles, [que] anuncia la necesidad siempre urgente del cuerpo y da cuenta de su satisfacción provisional». ${ }^{26}$ Esta descripción del cuerpo destaca la dimensión pre-reflexiva de la existencia corporal. Un dolor repentino, tomemos por caso un golpe en el dedo chico del pie contra un mueble mal ubicado, interrumpirá la tediosa tarea de acomodar los papeles que tengo sobre mi mesa requiriendo ahora toda mi atención. Sin dudas, mi atención puede volver rápidamente sobre los papeles desordenados, pero mi comportamiento estará dedicado a soportar el dolor en principio punzante y opresivo que irá convirtiéndose en un incómodo hormigueo.

El movimiento del cuerpo, indisimulable la mayor de las veces, acompaña esta irrupción del dolor. Incluso cuando puedo soportar el dolor y sostener una postura habitual, como en el caso de un dolor de muelas constante, la irradiación de ese dolor provocará que mi mandíbula se contracture, que coma de un solo lado de la boca, que evite hablar, etc. Mi comportamiento, aun mínimamente, cambiará y mi percepción del mundo se encontrará a merced del capricho del aparecer y desaparecer de la

25. Jean-Paul Sartre propone una noción de espacio igualmente interesante que denomina espacio hodológico, término que recupera de la obra del psicólogo gestáltico K. Lewin. Tal concepción de espacio involucra concebir al mismo como el campo de significaciones que abre el para-sí como proyecto. Cf. -Sartre, J.P.: (1943) El Ser y la Nada; Ed. Altaya; Barcelona, 1996. Traducción de Juan Valmar, p. 335 y ss.

26. Ibidem, p. 185.

Thémata. Revista de Filosofía No54 (2016) pp.: 175-190. 
dolencia. Los cambios posturales en muchos casos pueden modificar las intensidades de la dolencia y apaciguar el requerimiento atencional que el cuerpo reclama a la conciencia reflexiva.

A partir de lo dicho en esta sección y de las consideraciones realizadas en las secciones anteriores, puedo afirmar que la línea de investigación que coloca al cuerpo como sujeto de experiencia del dolor permite una lectura de la relación entre atención y cuerpo a partir de la noción de esquema corporal y de cuerpo en movimiento. Si entendemos al dolor como un fenómeno vivido por el cuerpo, deshaciéndonos de las investiduras dualistas, se patentiza el hecho de que la relación de la conciencia y el mundo es una relación vehiculizada por la corporeidad. Ya no alcanza con suponer que la conciencia se vuelve hacia una manifestación, dolorosa en este caso, de su existencia corpórea, sino que precisamente por su existencia corpórea, es que el mundo vivido puede ser puesto entre paréntesis ante la aparición brusca de una vivencia como es la del dolor.

\section{Consideraciones sobre la pertenencia de la fenomenolo- gía del dolor a la fenomenología de la corporeidad}

Si la experiencia del dolor físico resulta un caso particular de análisis de un fenómeno corporal, pues se concibe en términos de vivencia fundamentalmente encarnada, entonces el caso del dolor se deja esclarecer a partir de lo que se ha denominado Fenomenología de la corporeidad. Sin embargo, ambos enfoques, el de Serrano de Haro y el de la perspectiva merleau-pontyana, dan cuenta de dos nociones distintas de cuerpo o de corporeidad. Por esta razón, es conveniente preguntar ¿qué se entiende por cuerpo en cada caso?

El análisis de Sarrano de Haro parte desde el punto de vista de que la conciencia tiene una importancia radical respecto al cuerpo, que es un objeto para ella. Esto es así ya que la conciencia guarda una relación constituyente con el cuerpo que acentúa un dualismo mesurado, o al menos privilegia el papel activo de la conciencia con relación al objeto pasivo cuerpo. En los análisis fenomenológicos de Serrano de Haro, el cuerpo conserva el lugar de compañero íntimo de la conciencia pero no se identifica con ella. Para el autor, siguiendo en esto a Husserl, el cuerpo (cuerpo propio) es un aquí absoluto o aparecer cero de la experiencia. ${ }^{27} \mathrm{Y}$ esta manera de entender al cuerpo como centro absoluto del aquí y ahora implica, para la fenomenología husserliana, comprenderlo como reposo o quietud, «de modo

27. Cfr. Serrano de Haro, A. "Fundamentación para una fenomenología del cuerpo" en La posibilidad de la fenomenología, Madrid, Ed. Complutense, 1997, p. 210.

Thémata. Revista de Filosofía No54 (2016) pp.: 175-190. 
parecido como el árbol reposa aunque sus ramas se muevan». ${ }^{28} \mathrm{El}$ cuerpo que por su estructura es un "más acá" de las direcciones orientadas, sin perspectiva sobre sí mismo, «mantiene con el yo una relación que no es ni de identidad objetiva (unidad) ni de disparidad objetiva (separabilidad, dualidad de cosas), sino de referencia mutua e irreductible en el seno de la única vida intencional a que ambos pertenecen y en que ambos han surgido» ${ }^{29}$.

A pesar del reconocimiento del lugar fundamental de la corporeidad para la vida de la conciencia, la distancia entre conciencia y cuerpo no es un problema para la perspectiva husserliana. Mientras que, para la perspectiva merleau-pontyana concebir al cuerpo solo como centro de referencias o aquí y ahora absoluto, no hace del cuerpo más que una masa pesada y obscura apenas identificable: «el cuerpo por sí mismo, el cuerpo en reposo no es más que una masa oscura, lo percibimos como un ser preciso e identificable cuando se mueve hacia las cosas, cuando se proyecta intencionalmente hacia el exterior, y entonces sólo está en el rabillo del ojo y al margen de la conciencia, cuyo centro está ocupado por las cosas y por el mundo". ${ }^{30}$ De modo que el cuerpo no puede ser constituido como objeto por la conciencia, ya que, en el enfoque merleau-pontyano, él mismo es la estructura originaria, una intencionalidad motriz dirigida hacia el mundo, desde el mundo y por el mundo. Es más, en el análisis merleau-pontyano, el cuerpo no es nunca un mero mecanismo o un organismo funcional, pero tampoco es un objeto intencional. En este sentido, para dar cuenta de un sujeto que es su cuerpo, Merleau-Ponty adopta el término de conciencia encarnada-encarnada a un cuerpo que si bien es el cuerpo vivido del que también Husserl hablaba, no es un objeto constituido. Los supuestos husserlianos se modifican y se adoptan los de una conciencia corporal y una intencionalidad operante o motriz ligada a la carnalidad y asociada a la propia capacidad de cinestesias y cenestesias. La percepción del mundo se asocia a la capacidad motriz del cuerpo propio, modificando y organizando las relaciones intencionales que el cuerpo establece con su situacionalidad a partir del movimiento. En el caso del dolor, esta relación del "yo puedo" (o intencionalidad motriz) con el mundo guarda un vínculo especial, pues, ante la aparición repentina del dolor, el mundo cambia su tonalidad habitual, y es mi propia corporeidad la que debe reajustarse al requerimiento del mundo. Este horizonte congelado, abandonado un instante por la in-

28. Referencia a Husserl (H XV, 263-264) en Serrano de Haro, A. "Fundamentación para una fenomenología del cuerpo" en La posibilidad de la fenomenología, Madrid, Ed. Complutense, 1997, p. 211.

29. Serrano de Haro, A. "La precisión del cuerpo. Análisis filosófico de la puntería", Ed. Trotta, Madrid, 2007, p. 61.

30. Merleau-Ponty, M. Fenomenología de la percepción, F.C.E., Mexico-Buenos Aires, 1957, p. 356-357.

Thémata. Revista de Filosofía №54 (2016) pp.: 175-190. 
minente presencia dolorosa, me solicita, el mundo requiere mi volver a poner el cuerpo en dirección a él, en palabras de Merleau-Ponty: «El cuerpo propio está en el mundo como el corazón en el organismo: mantiene constantemente con vida el espectáculo visible, lo anima y lo alimenta interiormente, forma un sistema con él $»^{31}$

$* * *$

Aún a pesar de la distancia teórica de estas dos perspectivas de análisis del dolor, el hecho de que en los dos casos el cuerpo sea el sujeto de la experiencia doliente justifica la necesidad de aceptar, por el momento, ambas descripciones. Si bien la fenomenología de la corporeidad cuenta con un espeso conjunto de investigaciones y desarrollos, la fenomenología del dolor físico se encuentra en plena juventud y no ha ganado, del todo, independencia de los estudios generales sobre el cuerpo. Incluso, en muchos casos, el dolor es solo un ejemplo en la literatura sobre el cuerpo. Pero a diferencia de muchos otros fenómenos analizados, el caso del análisis del dolor físico constituye no solo un elemento fundamental para comprender y describir la encarnación de la conciencia, sino un punto nodal para el intercambio con otras disciplinas que se ven interpeladas por la naturaleza compleja del dolor.

\section{Referencias bibliográficas}

Arregui J. V. "Cenestesia y cuerpo vivido. ¿Por qué Marcel abandonó sus primeras formulaciones sobre el cuerpo sujeto?" Daimon. Revista de Filosofía, n.32, 2004, 145-158.

Choza J. "Dimensiones antropológicas del dolor", Anuario Filosófico, vol. 10, n² 2, 1997, p. 39-57. ISSN 0066-5215.

Depraz, N. "When Trascendental Genesis Encounters the Naturalization Project". Naturalizing Phenomenology. Issues in Contemporary and Cognitive Science, Petitot, J., Varela, F., Pachoud, B. \& Roy, J. M. (eds.). Stanford, CA: Stanford University Press, 1999. 464-489.

Gallagher, S."Body image and body schema: A conceptual clarification" en Journal of Mind \& Behavior, Autumm, 1986, Volumen 7, Number 4, pp 451-554.

Geniusas, S. "On naturalism in pain research: a phenomenological critique", Metodo. International Studies in Phenomenology and Philosophy, 2013, Vol. vol. 1, n. 1.

31. Ibidem, p. 223.

Thémata. Revista de Filosofía N54 (2016) pp.: 175-190. 
Geniusas, S. "The origins of the phenomenology of pain: Brentano, Stumpf and Husserl", Continental Philosophy Review, Vol. 47, Issue 1, pp. $1-17,2014$.

Husserl, E.: Investigaciones Lógicas, Revista de Occidente, Madrid, Trad. M. García Morente y J. Gaos, 1967.

Husserl, E.: Ideas relativas a una fenomenología pura y a una filosofía fenomenológica, Fondo de Cultura Económica, México, 1986. Traducción: José Gaos.

Landgrebe, L., "Principios de la teoría de la sensación", en El camino de la Fenomenología, Ed, Sudamericana, Buenos Aries 1963, p. 172-191.

Leder, D. The absent body, The Univessity of Chicago Press, Chicago, United States of America, 1990.

López Sáenz, C. "Hermenéutica del cuerpo doliente-dolido desde la fenomenología del sentir" en Investigaciones Fenomenológicas, Vol. monográfico 2: "Cuerpo y alteridad", 2010.

Merleau-Ponty, M. Fenomenología de la percepción, F.C.E., Mexico-Buenos Aires, 1957, Traducción: Emilio Uranga.

Ramachandran, V. S. - Blackeslee, S. Phantoms in the Brain, William Morrow and Co., New York, 1998.

Sartre, J.P.: (1943) El Ser y la Nada; Ed. Altaya; Barcelona, 1996. Traducción: Juan Valmar.

Serrano de Haro, A. A propósito de la fenomenología del dolor, Crítica, Año 62, nº 981, 2012.

Serrano de Haro, A. La precisión del cuerpo. Análisis filosófico de la puntería, Ed. Trotta, Madrid, 2007.

Serrano de Haro, A. "Defensa de la perspectiva fenomenológica en el análisis del dolor" en Pensar la compasión, Ed. Universidad Pontifica Comillas, Reflexiones Comillas, Filosofía, 1, Año 59, nº 959, 2009. P 161-172.

Serrano de Haro, A. "Atención y dolor. Análisis Fenomenológico" en José Ortega y Gasset/José Gaos/Joaquín Xirau/ Leopoldo-Eulogio Palacios/Agustín Serrano de Haro, Cuerpo vivido. Madrid, Encuentro, 2010.

Serrano de Haro, A. "Fundamentación para una fenomenología del cuerpo" en La posibilidad de la fenomenología, Madrid, Ed. Complutense, 1997, pp 185-216. 